\title{
Essere in controllo o avere sotto controllo?
}

\section{Giuseppe Patota}

PUBBLICATO: 14 MAGGIO 2019

\section{Quesito:}

I nostri lettori ci sottopongono l'espressione essere in controllo usata, secondo qualcuno dai commentatori calcistici, in luogo di avere il controllo (della situazione); ipotizzano anche la derivazione dall'inglese to be in control.

\section{Essere in controllo o avere sotto controllo?}

espressione essere in controllo è certamente un calco dell'inglese to be in control, che i dizionari bilingui inglese-italiano traducono 'tenere, avere sotto controllo'. D'altra parte, nessun vocabolario della nostra lingua ha mai registrato, almeno finora, essere in controllo con questo significato.

A questi due dati se ne aggiunge un terzo: molte espressioni in cui è presente la sequenza essere in (per esempio: essere in pace, essere in tempo, essere in anticipo, essere in ritardo, ecc.) rinviano, per l'appunto, a un essere più che a un agire. Perciò, chi senta dire essere in controllo ha tutte le ragioni di interpretare "essere sotto controllo" e non "tenere, avere sotto controllo".

Per tenere sotto controllo il proprio italiano, dunque, è meglio non dire mai di "esserne in controllo".

Nota bibliografica:

- Grande dizionario Hazon di inglese, Milano, Garzanti Linguistica, 20I7

- Il Nuovo Treccani, diretto da Valeria Della Valle e Giuseppe Patota, Roma, Istituto della Enciclopedia Italiana, 20I8

- Il Ragazzini 2019. Dizionario inglese-italiano, italiano-inglese, Bologna, Zanichelli, 2018

\section{Cita come:}

Giuseppe Patota, Essere in controllo o avere sotto controllo?, "Italiano digitale", 2019, IX, 2019/2 (aprile-giugno)

DOI: $10.35948 / 2532-9006 / 2020.3114$

Copyright 2019 Accademia della Crusca

Pubblicato con licenza creative commons CC BY-NC-ND 